\title{
Sinus Histiocytosis with Massive Lymphadenopathy (Rosai-Dorfman Disease): A Case Report and Literature Review
}

\author{
Rabindra Bhakta Pradhananga ${ }^{1}$ Kripa Dangol ${ }^{1}$ Anjan Shrestha ${ }^{2}$ Dharma Kanta Baskota ${ }^{1}$ \\ ${ }^{1}$ Department of ENT-Head and Neck Surgery, Tribhuvan University \\ Address for correspondence Rabindra Bhakta Pradhananga, MBBS, MS \\ (ENT- Head \& Neck Surgery), Department of ENT-Head \& Neck Surgery, \\ Teaching Hospital, Kathmandu, Nepal \\ 2 Department of Pathology, Tribhuvan University Teaching Hospital, \\ Tribhuvan University Teaching Hospital, Kathamandu +977 , Nepal \\ Kathmandu, Nepal \\ (e-mail: rabindrabp@yahoo.com; rabindra9034@gmail.com).
}

Int Arch Otorhinolaryngol 2014;18:406-408.

\begin{abstract}
Keywords

- sinus histiocytosis

- massive lymphadenopathy

- Rosai-Dorfman disease

Introduction Rosai-Dorfman disease (RDD) is a rare histiocytic proliferative disorder of unknown etiology. Usually it presents with massive painless cervical lymph node enlargement. Histologically, it shows proliferation of distinctive histiocytic cells that demonstrate emperipolesis in the background of a mixed inflammatory infiltrates. Immunohistochemically, the cells are positive for markers such as CD68 and S100.

Objective To report a case of a 12-year-old patient with multiple sites of cervical lymphadenitis, which was diagnosed as RDD histopathologically as well as immunohistologically.

Resumed Report A 12-year-old girl presented with multiple painless sites of cervical lymphadenitis without any systemic and other ear, nose, and throat manifestations. The biopsy report of the lymph node showed dilatation of the sinuses, filled with histiocytes having foamy cytoplasm. Many of the histiocytes were engulfing mature lymphocytes. The sinus histiocytes were strongly positive for S-100 protein.

Conclusion RDD must be considered in the differential diagnosis of massive or multiple lymphadenopathies.
\end{abstract}

\section{Introduction}

Sinus histiocytosis with massive lymphadenopathy (SHML), which is also known as Rosai-Dorfman disease (RDD), is a rare histiocytic proliferative disorder of unknown etiology initially described by Rosai and Dorfman in 1969. ${ }^{1}$ RDD can occur in any age group but is most commonly seen in children and young adults. ${ }^{2}$ Usually it presents with massive painless lymph node enlargement at any site, with the cervical nodes being common. Histologically, lymph nodes show pericapsular fibrosis and dilated sinuses, heavily infiltrated with large histiocytes, lymphocytes, and plasma cells. ${ }^{1,3}$ Immunohistochemically, the sinus histiocytes are strongly positive for S-100 protein. ${ }^{4}$

received

April 10, 2014

accepted

June 29, 2014

published online

August 25, 2014
DOI http://dx.doi.org/

10.1055/s-0034-1387163. ISSN $1809-9777$.
Though various modes of treatment like chemotherapy, radiotherapy, and surgical debulking have been tried, all have shown limited efficacy. The course of the disease is somewhat variable, from complete spontaneous remission in some cases to protracted clinical disease for years in other cases. The possibility of involvement of vital organs leading to death is also mentioned in some cases.

\section{Review of Literature with Differential Diagnosis}

RDD commonly presents with massive bilateral and painless cervical lymphadenopathy with fever, night sweating, and weight loss. Extranodal involvement has been documented in

Copyright $\odot 2014$ by Thieme Publicações License terms Ltda, Rio de Janeiro, Brazil

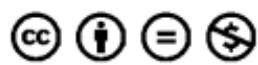


25 to $40 \%$ of cases, and organs such as skin, respiratory tract, reticuloendothelial system, genitourinary tract, and thyroid are commonly involved.

Ju et al reported a case of RDD in a 26-year-old man with cervical and mediastinal lymphadenopathy with pleural effusion in 2009. ${ }^{5}$ Agarwal et al reviewed seven cases (five nodal and two extranodal) of RDD. Of these, five patients were followed. ${ }^{6}$ However, four had stable disease, and one developed histiocytic sarcoma after a gap of 4 years. Zhu et al found more purely extranodal RDD involving the central nervous system on retrospectively analyzing 13 cases of RDD. $^{7}$

Clinically, the nodal type of RDD should be differentiated from lymphoma and any other causes of chronic lymphadenitis, such as tubercular lymphadenitis, Kikuchi-Fujimoto disease, among others. Histologically, the disease must be differentiated from Langerhans cell histiocytosis and infectious and lymphoproliferative disorders as well as sinus hyperplasia. S-100 positivity can usually distinguish between the latter condition and RDD, whereas in both conditions the histiocytes have a strong macrophage antigen expression. ${ }^{4}$

\section{Case Report}

A 12-year-old girl visited the ENT \& Head and Neck Surgery Out Patient Department with presentation of bilateral multiple painless swelling in neck of 2 months' duration. She also complained of mild to moderate fever and weakness. Her general physical condition looked normal.

Examination of the neck revealed enlarged lymph node on both sides ( $\mathbf{- F i g s . ~} 1$ and $\mathbf{2}$ ). Left side examination noted level II $\left(3 \times 4 \mathrm{~cm}^{2}\right)$, level III $\left(2 \times 1 \mathrm{~cm}^{2}\right)$, and level V $\left(3 \times 3 \mathrm{~cm}^{2}\right)$

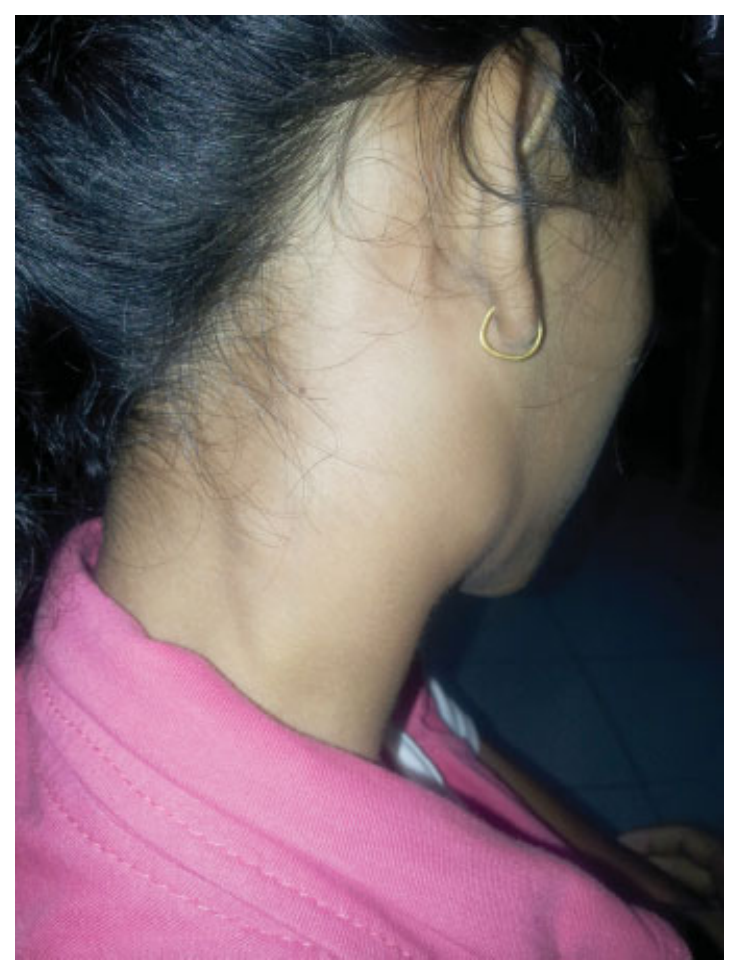

Fig. 1 Multiple massive cervical lymphadenopathy on right side.

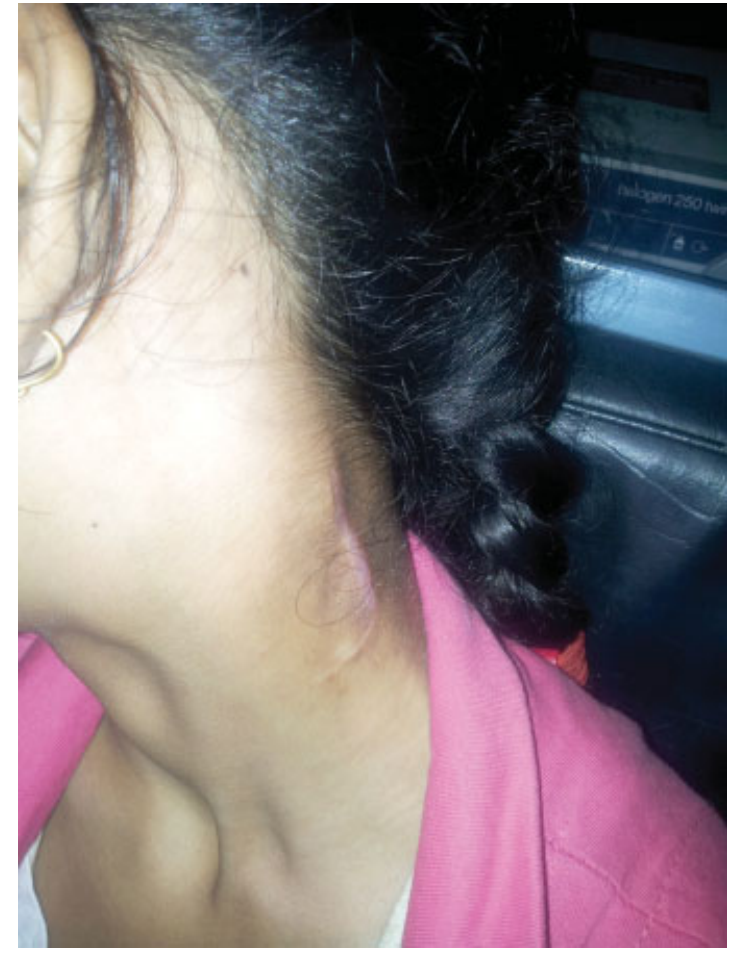

Fig. 2 Cervical lymphadenopathy with scar on left side of neck.

lymph nodes palpable with scar at a previous biopsy site (taken somewhere else). Similarly, the right side revealed level IB $\left(3 \times 3 \mathrm{~cm}^{2}\right)$, level III $\left(1 \times 1 \mathrm{~cm}^{2}\right)$, and level V $(4 \times 3$ $\mathrm{cm}^{2}$ ) lymph nodes. All the lymph nodes were found to be firm, well defined, nontender, and mobile. Examination of ear, nose, oral cavity, oropharynx, and larynx revealed normal findings.

Routine blood investigations revealed polymorphonuclear leukocytosis and increased erythrocyte sedimentation rate $(55 \mathrm{~mm} / \mathrm{h})$. Fine Needle Aspiration Cytology (FNAC) was inconclusive. Ultrasonography (USG) of the neck showed multiple enlarged lymph nodes in both sides of the neck, suspicious of lymphoma. USG of the abdomen and pelvis showed normal findings.

Because a previous biopsy taken elsewhere was inconclusive, a revision biopsy was taken and sent for histopathologic examination. Histopathology showed a gross appearance of well-encapsulated brownish tissue measuring $1.8 \times 1.5 \mathrm{~cm}^{2}$, and cut sections showed homogenous white areas. Microscopic examination of the sections of lymph node showed dilatation of the sinuses, filled with histiocytes having foamy cytoplasm. Many of the histiocytes were seen engulfing mature lymphocytes (emperipolesis; -Figs. 3 and $\mathbf{4}$ ). The histopathologic diagnosis was RDD, which was further confirmed by immunohistochemistry. The histiocytic cells were S-100-positive.

The patient was started on oral prednisolone in tapering dose with continuation of low dose for 1 month. Her condition was improved at first follow-up after 1 week, and she will be followed further. 


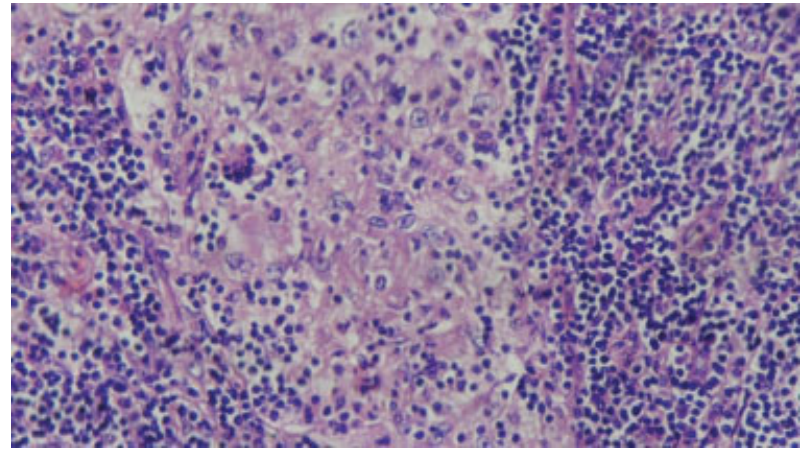

Fig. 3 Section with hematoxylin and eosin stain under $20 \times 100$ magnification: dilated sinuses with histiocytes.

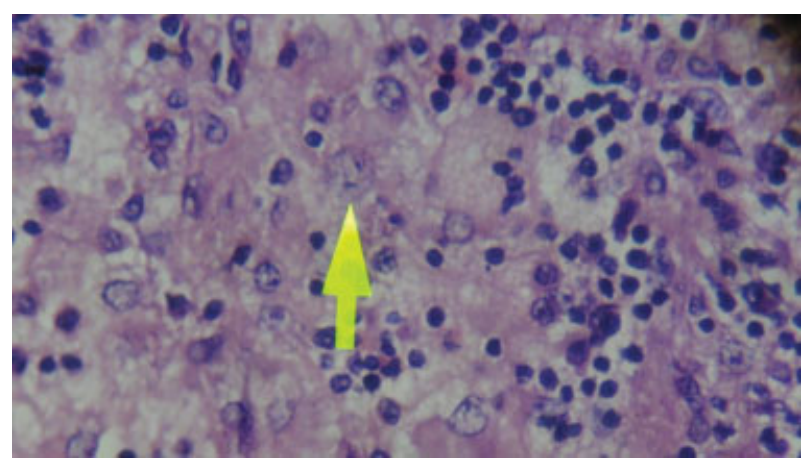

Fig. 4 Section with hematoxylin and eosin stain under $40 \times 100$ magnification: characteristic emperipolesis.

\section{Discussion}

RDD is a rare, idiopathic, histiocytic proliferative disorder. Consequently, many characteristics of this disease remain unknown, restricting early diagnosis and proper treatment. Various theories have been implicated as an etiology; however, there is no strong evidence for any of them.

The presentation of the disease also varies with site of involvement. Commonly it presents with massive cervical lymphadenopathy. But extranodal involvement has also been documented in around $40 \%$ of cases, the most common being skin followed by upper respiratory tract and bone. ${ }^{8}$ However, RDD also can occur in a variety of other sites, including the genitourinary system, lower respiratory tract, oral cavity, and soft tissues. ${ }^{9}$ Other reported specific sites include brain, spine, liver, kidney, thyroid, breast, parotid gland, orbit, nasal cavity, and lungs.

The characteristic pathologic feature of this disease is proliferation of distinctive histiocytic cells that demonstrate emperipolesis (the engulfment of lymphocytes and erythrocytes by histiocytes) in the background of a mixed inflammatory infiltrate, consisting of moderately abundant plasma cells and lymphocytes. ${ }^{3}$ Immunohistochemically, SHML cells express phagocytic markers such as CD68 and S100, but not the markers for Langerhans (CD1a) or dendritic cells (DRC, CD23, and CNA42). ${ }^{2}$

Pulsoni et al reviewed case reports of 80 patients who received various modes of treatments. ${ }^{9}$ The study showed all of the treatment modalities including chemotherapy and radiotherapy were ineffective; however, surgical debulking when required was effective. Spontaneous resolution of adenopathies is frequently observed so that clinical observation without treatment is advisable when possible. Systemic corticosteroids are usually helpful in decreasing nodal size and symptoms; however, they can be quite immunosuppressive and RDD lesions can recur after a short period of interruption. ${ }^{9}$ Although Oka et al reported an effective prolonged course of low-dose prednisolone in a case of RDD with skin and lymph node involvement with respiratory obstruction, its role cannot be generalized. ${ }^{10}$

\section{Final Comments}

RDD must be considered in the differential diagnosis of young patients who exhibit massive or multiple lymphadenopathies, especially when involvement of the cervical area occurs. The disease can be diagnosed on the histopathologically and immunohistochemically.

\section{References}

1 Rosai J, Dorfman RF. Sinus histiocytosis with massive lymphadenopathy. A newly recognized benign clinicopathological entity. Arch Pathol 1969;87(1):63-70

2 Bernácer-Borja M, Blanco-Rodríguez M, Sanchez-Granados JM, Benitez-Fuentes R, Cazorla-Jimenez A, Rivas-Manga C. Sinus histiocytosis with massive lymphadenopathy (Rosai-Dorfman disease): clinico-pathological study of three cases. Eur J Pediatr 2006; 165(8):536-539

3 Juskevicius R, Finley JL. Rosai-Dorfman disease of the parotid gland: cytologic and histopathologic findings with immunohistochemical correlation. Arch Pathol Lab Med 2001;125(10): $1348-1350$

4 Eisen RN, Buckley PJ, Rosai J. Immunophenotypic characterization of sinus histiocytosis with massive lymphadenopathy (Rosai-Dorfman disease). Semin Diagn Pathol 1990;7(1):74-82

$5 \mathrm{Ju}$ J, Kwon YS, Jo KJ, et al. Sinus histiocytosis with massive lymphadenopathy: a case report with pleural effusion and cervical lymphadenopathy. J Korean Med Sci 2009;24(4):760-762

6 Agarwal A, Pathak S, Gujral S. Sinus histiocytosis with massive lymphadenopathy-a review of seven cases. Indian J Pathol Microbiol 2006;49(4):509-515

7 Zhu F, Zhang JT, Xing XW, et al. Rosai-Dorfman disease: a retrospective analysis of 13 cases. Am J Med Sci 2013;345(3):200-210

8 Foucar E, Rosai J, Dorfman R. Sinus histiocytosis with massive lymphadenopathy (Rosai-Dorfman disease): review of the entity. Semin Diagn Pathol 1990;7(1):19-73

9 Pulsoni A, Anghel G, Falcucci P, et al. Treatment of sinus histiocytosis with massive lymphadenopathy (Rosai-Dorfman disease): report of a case and literature review. Am J Hematol 2002;69(1):67-71

10 Oka M, Kamo T, Goto N, et al. Successful treatment of RosaiDorfman disease with low-dose oral corticosteroid. J Dermatol 2009;36(4):237-240 